\title{
Big data services drive mobile crowd embedded opportunistic control mechanism for biological engineering
}

\author{
Hai-Chao Wang ${ }^{1,2^{*}}$ and Zeng Dong ${ }^{1}$
}

\begin{abstract}
Big data of biological engineering and mobile control increase the complexity of system control. In order to resolve the above problems and improve biological engineering system performance, this paper proposes a large data-driven and mobile crowd embedded opportunistic control mechanism. First of all, the measurement model of established big data-driven biological embedded engineering was proposed based on the research of biological engineering with non-linear and unpredictability. Based on the characteristics of the mobile crowd terminal perception outside interference, we proposed the mobile crowd biological engineering optimization opportunities control mechanism. The experimental results show that the established control mechanism in a long-term, large-scale performance still has high performance and high temperature. In addition, under different pressure, the rate of convergence of the scheme established is superior to the biological engineering control scheme based on coordination control.
\end{abstract}

Keywords: Biological engineering, Embedded systems, Opportunistic control, Big data services drive, Mobile crowd

\section{Introduction}

How to combine biological engineering with computer technology, software engineering, and a control engineering application has become a research hotspot in recent years [1]. At the same time, biological engineering multi-objective optimization [2] has gradually brought many challenges, such as the control strategy of biological reaction resistance to interference [3]. It has become the research key issue that how to improve the control precision of biological engineering [4] and provide a performance guarantee for large-scale biological engineering [5].

Regarding biological big data, Nounou et al. used Wavelet-based multiscale filtering to mine the important features in measured biological data [6]. Stegmayer presented a novel integrated computational intelligence approach for biological data mining that involves neural networks and evolutionary computation [7]. Chatziioannou et al. presented the web-based

\footnotetext{
* Correspondence: haichaowwang@sina.com

${ }^{1}$ College of Biological and Food Engineering, Suzhou University, Suzhou 234000, China

${ }^{2}$ Engineering Technology Research Center of Characteristics of Planting and Seedling Production, Suzhou University, Suzhou 234000, China
}

grid application that could exploit grid infrastructures for distributed data processing and management through a generic, consistent, computational analysis framework [8]. Nguyen et al. presented an alternative conception for local data integration based on a hybrid flat file, a generic data model, and configuration rules [9]. Carpendale et al. studied biological data visualization [10].

Mobile computing is closely associated with biological response. Atakan et al. proposed the biological foraging-inspired communication algorithm for the energy-efficient and spectrum-aware communication requirements [11]. Lin proposed the wireless power transfer scheme for cell phones or other mobile communication devices and biological implications [12]. Liu et al. studied the biological characteristic authentication and multimedia signal fast encoding over 5G for improving the security level of the Internet [13]. Lin designed the wireless power transfer for mobile applications and health effects [14]. Tsompanas proposed a CA model used as a virtual, easy-to-access, and bio-mimicking laboratory emulator, which would economize large time periods [15]. 
Regarding the relation of system control and biological engineering, Qian et al. analyzed and simulated an infinite-horizon optimal feedback control model, with linear plants, that contains both controldependent and control-independent noise and optimizes steady-state accuracy and energetic costs per unit time [16]. Chowdhury et al. demonstrated the effectiveness of the approach by transporting a yeast cell using four different types of gripper formations along collision-free paths on our OT setup. We analyzed the performance of the proposed gripper formations with respect to their maximum transport speeds and the laser intensity experienced by the cell that depends on the laser power used [17]. Yao et al. proposed a newly developed all-solid-state nanosecond pulse generator based on the Marx generator concept for this application [18]. Chen et al. presented a novel approach for the automated transportation of multiple cells by using robotically controlled holographic optical tweezers [19]. Nakano et al. discussed the issues concerned with transmission rate control in molecular communication, an emerging communication paradigm for bio-nan machines in an aqueous environment [20].

Based on the results of the above research, we proposed the big data services drive mobile crowd embedded opportunistic control mechanism for biological engineering.

The rest of the paper is organized as follows. "Section 2" describes the big data-driven biological embedded engineering measurement model. In "Section 3 ," we design the mobile crowd biological engineering opportunistic optimization control mechanism. Performance evaluations are given in "Section 4." We conclude the paper in "Section 5."

\section{Big data-driven biological embedded engineering measurement model}

Biological engineering is non-linear and unpredictable, and is composed of a series of large-scale physical reactions and the coupling reaction. Biological engineering of large data sources is an iterative cell. There are some problems in a survey of biological engineering, such as the non-linear objects, the coupling of data, and timevarying data source problems. In real-time measurements of biological engineering, the characteristics of the embedded outside objects would be obtained by measuring the object's environment in the measurement model.

Therefore, we designed an embedded project measurement model. The model can obtain the bioengineering embedded external factors and the influence of the normal reaction of biological engineering. The impact model have to obtain the reverse metabolism energy consumption of embedded biological.
We have therefore designed an embedded biological engineering piece of equipment. The equipment outside of biological data should embed the required amount of energy. The device can obtain a biological engineering survey object scale. The equipment size and embedded objects of the reproduction life cycle would be compared by measuring the parameters of biological engineering. According to the measurement parameters, embedded devices for biological embedded engineering guarantee stability. There are some peripheral terminal interfaces in the embedded devices. These interfaces are used to establish the high coupling reaction environment for the intrinsic biological reproduction. The biological response module was designed in the embedded equipment.

Embedded devices meet the conditions as shown in Fig. 1. The equipment added some buffer function of the embedded terminal. This embedded terminal can sense the driving factors of the external environment of biological engineering. These driving factors include embedded species, embedded data volume and biological reaction cycle, embedded device antiinterference ability, etc.

In order to weaken the biological engineering data source degeneration, we designed a data buffer in the embedded device. At the same time, we put forward the principle of biological embedded engineering reaction conditions with the best match, such as equations (1), (2), and (3).

$$
d_{b}=\sqrt{\frac{\sum_{i=1}^{N} x_{i}}{x_{\max }-x_{\min }}}
$$

Here, $d_{b}$ is the embedded biological engineering response data. $\mathrm{X}$ denotes the iteration of the embedded data volume. $\mathrm{X}_{\max }$ denotes the largest amount of data. $\mathrm{X}_{\text {min }}$ denotes the smallest amount of data. $\mathrm{N}$ is the embedded biological scale of biological engineering.

$$
t_{b}=d_{b}\left|\frac{\pi t}{\cos (\omega t)}-\frac{\frac{3}{4} \pi t}{\sin \left(\omega t+\frac{1}{2} \pi t\right)}\right|^{-2 \alpha}
$$

Here, $t_{b}$ is the embedded biological engineering reaction cycle. $\alpha$ is the impedance coefficient of embedded devices. $\mathrm{W}$ is the intrinsic biological response module and embedded biological response module angle with the outside world. $\mathrm{T}$ is the iteration biological reaction cycle. $T_{i}$ is the biological iteration reaction cycle.

$$
i_{b}=\frac{1}{\alpha-1} \sum_{i=1}^{N}\left|t_{b}-t_{i}\right|^{2}
$$

Here, $\mathrm{i}_{\mathrm{b}}$ is embedded equipment ability of immunity. 


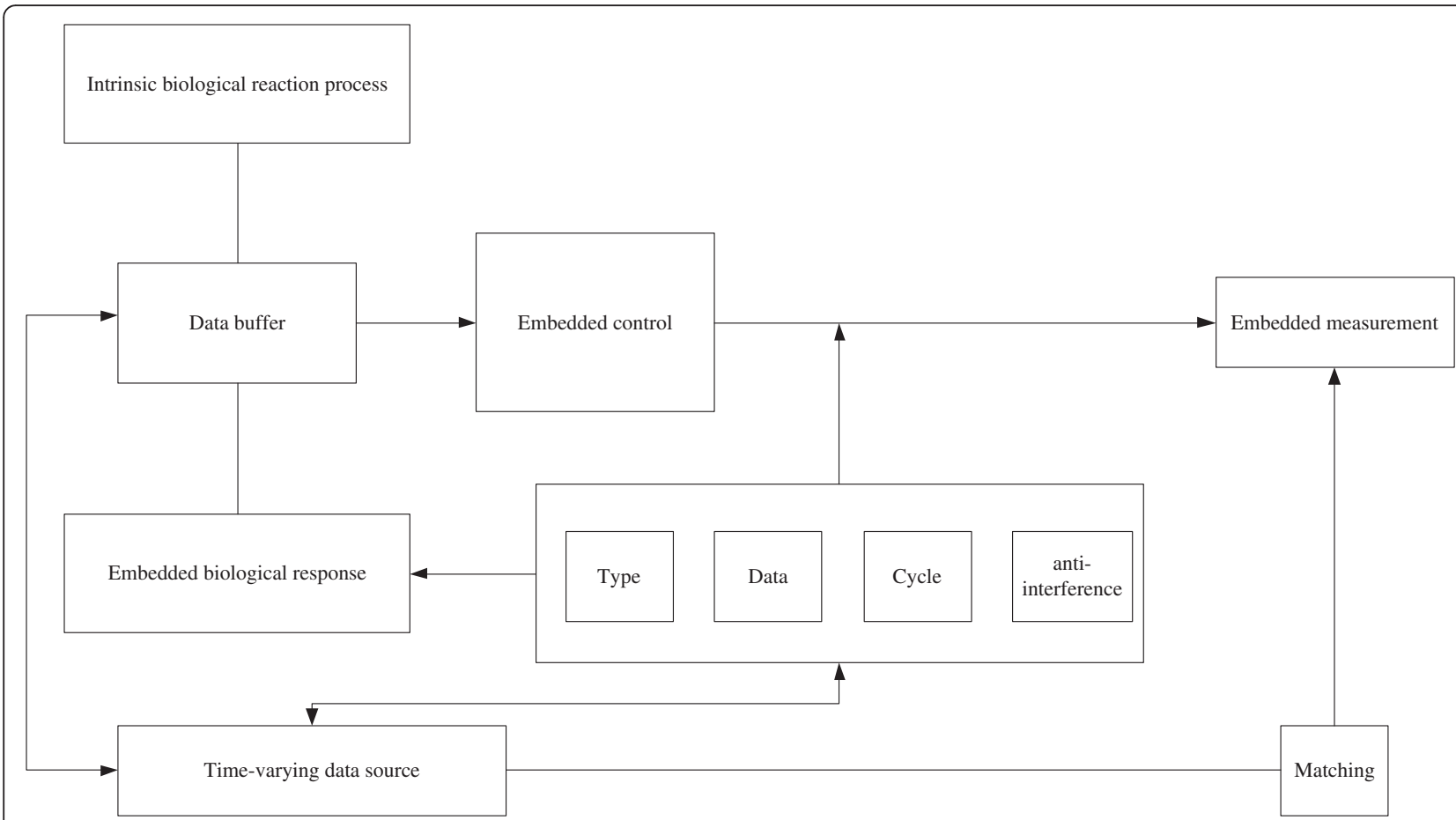

Fig. 1 Biological embedded device

Data buffer can effectively reduce the jitter of the data. For equations (1), (2), and (3) to establish the best matching principle can be a real-time perception biological engineering response to environmental changes. This principle can be applied to establish the effective matching between the data source and the data quantity.

The embedded equipment shown in Fig. 1 can ensure the normal response level of biological engineering and improve the time variability and the unpredictable nature of the data. When the data process and data size leveled off, the large data-driven bioengineering will further improve the quality of biological engineering. The measurement model of biological embedded engineering was constructed based on datadriven. $\mathrm{N}$ denotes the biological engineering response of $B_{M}$ vector, as shown in equation (4).

$$
\left\{\begin{array}{l}
B_{M}=\left\{\left(b_{1}, b_{2}, \cdots, b_{k}\right) \mid k=1,2, \cdots, u\right\} \\
u=\|N\|^{2}, k \geq v \\
u=\sqrt{N}, k<v
\end{array}\right.
$$

Here, $\mathrm{k}$ is the active biological response scale of sample points, $\mathrm{u}$ is embedded devices activated sample points, and $\mathrm{v}$ is the biological response data buffer factor for embedded devices.
Biological engineering embedded equipment feedback characteristics of big data are linear weighting of $L_{W}$, embedded weight $E_{W}$, and buffer weight $B_{W}$. The above big data characteristics have to meet the relationship shown in equation (5).

$$
\left\{\begin{array}{l}
B_{D C}=\left\{b_{D C} \mid b_{D C} \in\left(L_{W}, E_{W}, B_{W}\right)\right\} \\
L_{W} \cap E_{W}=\varnothing \\
R^{N} \rightarrow R^{u}
\end{array}\right.
$$

Here, $B_{D C}$ is the business data catalog comprehensive weight vector. The empty $\mathrm{L}_{\mathrm{W}}$ vector and $\mathrm{E}_{\mathrm{W}}$ vector intersection is embedded in order to ensure the equipment does not affect the big data linear transformation. The change from $R^{N}$ to $R^{\mathrm{u}}$ showed that $\mathrm{N}$ sample points of non-linear space would be mapping with the actual space biological response.

To sum up, the biological engineering big datadriven embedded measurement architecture is shown in Fig. 2.

\section{Mobile crowd biological engineering opportunistic optimization control mechanism}

In the biological engineering optimization method, we mainly considered the random interference factors on biological engineering and presented a biological engineering control mechanism based on mobile crowd and its architecture. The architecture can solve the 


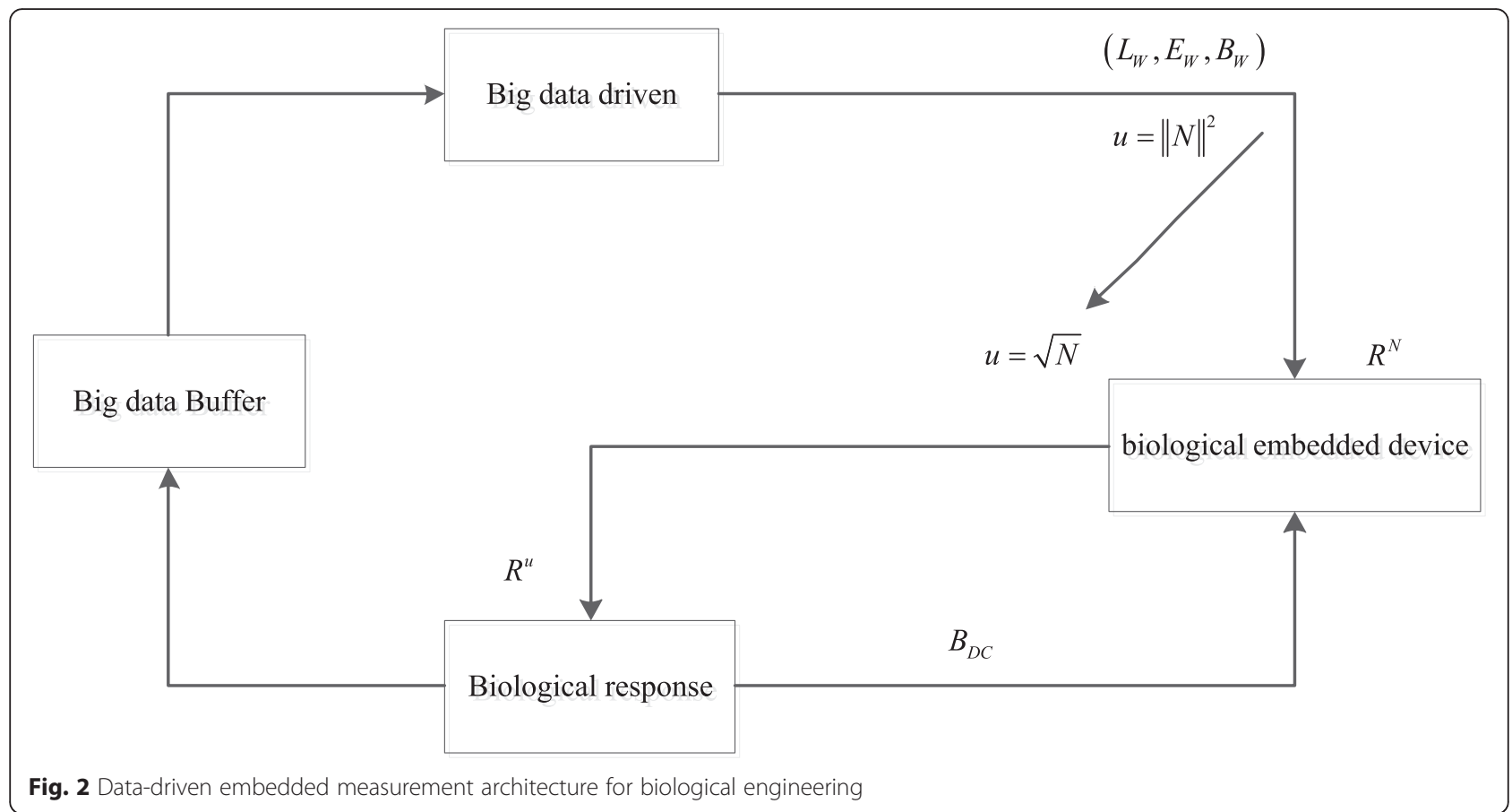

problem of the random interference and time variability of biological engineering by moving the crowd and big data.

The mobile crowd terminal inputs and outputs the parameters of mobile data model. The data model is used to define the control process of biological engineering. The data model of the sample set as shown in equation (6).

$$
\left\{\begin{array}{l}
D_{\text {input }}=\left\{d_{\text {input }} \mid \text { input } \in[m, n]\right\} \\
D_{\text {output }}=\left\{d_{\text {output }} \mid \text { output } \in[m, n]\right\} \\
d_{\text {input }} \in R^{m * n} \\
d_{\text {output }} \in R^{m * n} \\
\left\{\left(d_{\text {oinput }}, d_{\text {output }}\right) \mid \text { input }, \text { output } \in R\right\}
\end{array}\right.
$$

Here, $d_{\text {input }}$ is the input data parameters, $d_{\text {output }}$ is the output data parameters, $m$ is the data port number, $\mathrm{n}$ is the output port number, and $\mathrm{R}^{\mathrm{m} * \mathrm{n}}$ is the port matrix space.

Let $\mathrm{t}$ denote the data generation time of biological engineering. The non-linear calculation of mobile crowd data was shown in equation (7).

$$
\left\{\begin{array}{l}
y(t)=f\left(d_{\text {input }}, d_{\text {output }}\right) \\
y(t-k)=f\left(d_{\text {input }}, d_{\text {output }}\right) \sum_{i=1}^{k} y(i)
\end{array}\right.
$$

For the mobile crowd to find the best time to control biological engineering, the mobile crowd terminal and biological engineering data processing must meet the following conditions:

(1) To keep the mobile crowd nonlinear characteristics, the function $f\left(d_{\text {input }} d_{\text {output }}\right)$ have to be the Multi order Guide. The guide can be shown in equation (8).

$$
\left\{\begin{array}{c}
\frac{d(f)}{d t} \geq y_{\text {avg }} \\
\frac{d f}{d(t-k)} \geq y_{\text {avg }} \\
y_{\text {avg }}=\frac{\sum_{i=1}^{k} y_{i}}{k}
\end{array}\right.
$$

(2) The model of biological engineering in the matrix mapping relationship as shown in equations (9) and (10).

$$
\begin{aligned}
& \left.y(t)\right|_{t \rightarrow \infty}=\sum_{i=1}^{t} y_{i} T\left(\sqrt{\left(y_{i-1}, y_{i}\right)}\right) \\
& {\left[\begin{array}{ccc}
y_{1} & \cdots & y_{m} \\
y_{i-1} & \cdots & y_{m+i-1} \\
y_{t} & \cdots & y_{m+t-1}
\end{array}\right] T=\left[\begin{array}{c}
T\left(y_{1}, y_{2}\right) \\
T\left(y_{i}, y_{m+i-1}\right) \\
T\left(y_{t}, y_{m+t-1}\right)
\end{array}\right]}
\end{aligned}
$$

Here, the matrix $\mathrm{T}$ denotes the measurement model. (3) Biological response of mobile crowd wisdom kernel function as shown in equation (11). The kernel function must be non-linear after dealing with equation (12) to achieve perception of biological engineering control. 

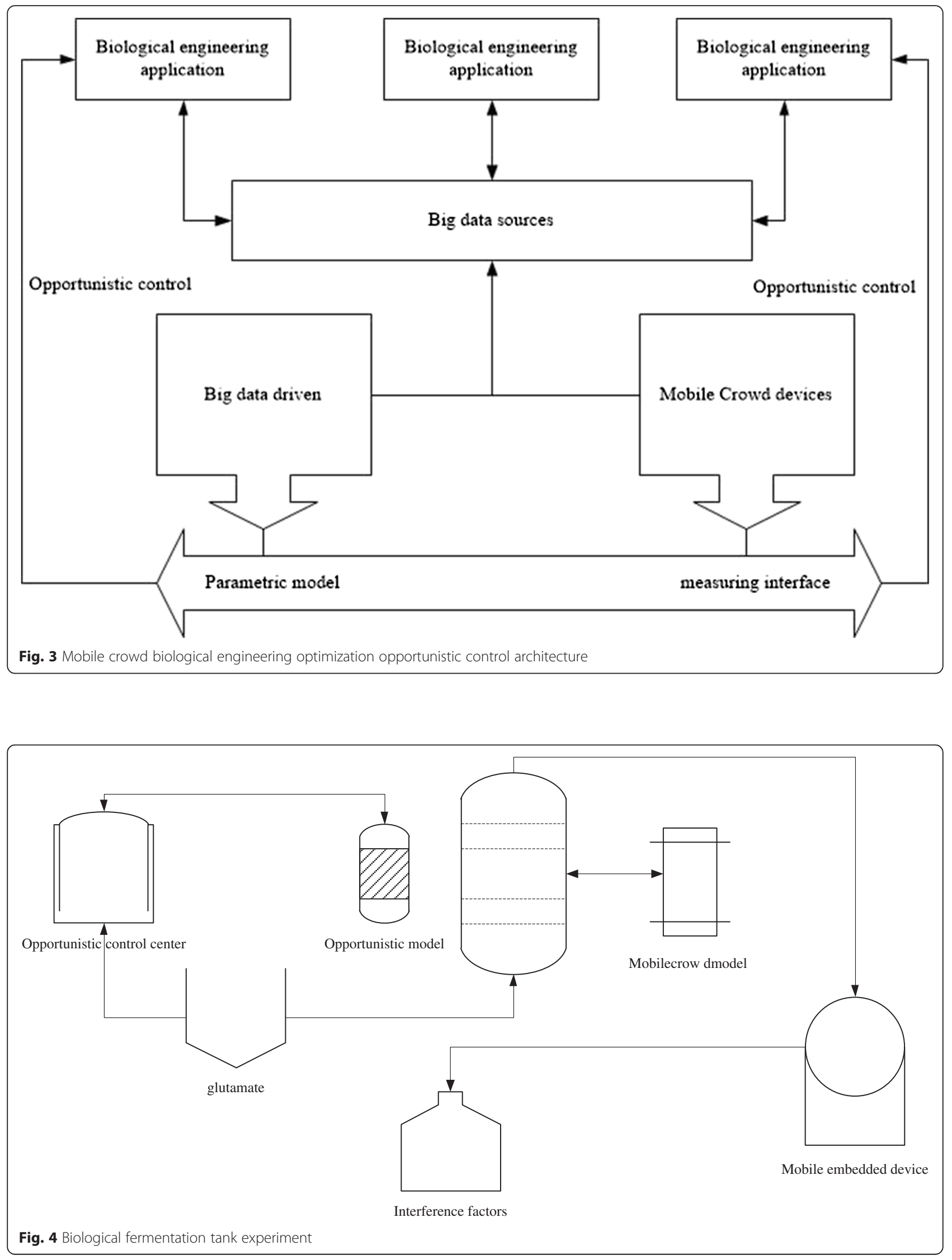
Table 1 Experiment settings

\begin{tabular}{|c|c|c|c|}
\hline Parameters & Value & Parameters & Values \\
\hline Tank capacity & $50 \mathrm{~L}$ & $\begin{array}{l}\text { Glutamic acid } \\
\text { dosage }\end{array}$ & $0.1-0.5 * 50 \mathrm{~L}$ \\
\hline Time & $28-50 \mathrm{~h}$ & Interference strains & Lysine \\
\hline Tank pressure & $0.01-0.1 \mathrm{Mpa}$ & $\begin{array}{l}\text { Experimental } \\
\text { temperature }\end{array}$ & $20-40{ }^{\circ} \mathrm{C}$ \\
\hline $\begin{array}{l}\text { Number of mobile } \\
\text { embedded devices }\end{array}$ & $2-5$ & Number of sample & 20 \\
\hline Sampling interval & $20 \mathrm{~min}$ & $\begin{array}{l}\text { Interference } \\
\text { frequency }\end{array}$ & $1-5$ per min \\
\hline $\begin{array}{l}\text { Opportunity control } \\
\text { weight }\end{array}$ & $0.2-0.8$ & $\begin{array}{l}\text { Interference } \\
\text { capacity }\end{array}$ & $0.2-0.8 \mathrm{~L}$ \\
\hline
\end{tabular}

$$
\begin{aligned}
& \left\{\begin{array}{l}
K_{B}=\left(\frac{\left\|y_{t}-y_{t-k}\right\|^{2}}{\delta} \sqrt{T_{t}}\right) \\
T_{x}=\left.y(x)\right|_{x \rightarrow t}
\end{array}\right. \\
& \left\{\begin{array}{c}
y(t)=\sum_{i=1}^{t} T\left(\sqrt{\left(y_{i-1}, y_{i}\right)}\right) K_{B} \\
K_{B} T_{x} \geq \sqrt{\left.y(t)\right|_{t \rightarrow \infty}}
\end{array}\right.
\end{aligned}
$$

To sum up, we can obtain the optimization control probability of the mobile crowd biological engineering such as equation (13). The weighting factor would be changed through optimization control probability. Biological engineering optimization would be linear by controlling the probability. The opportunistic selection progress would be non-linear with the linear optimization, as shown in equation (14).

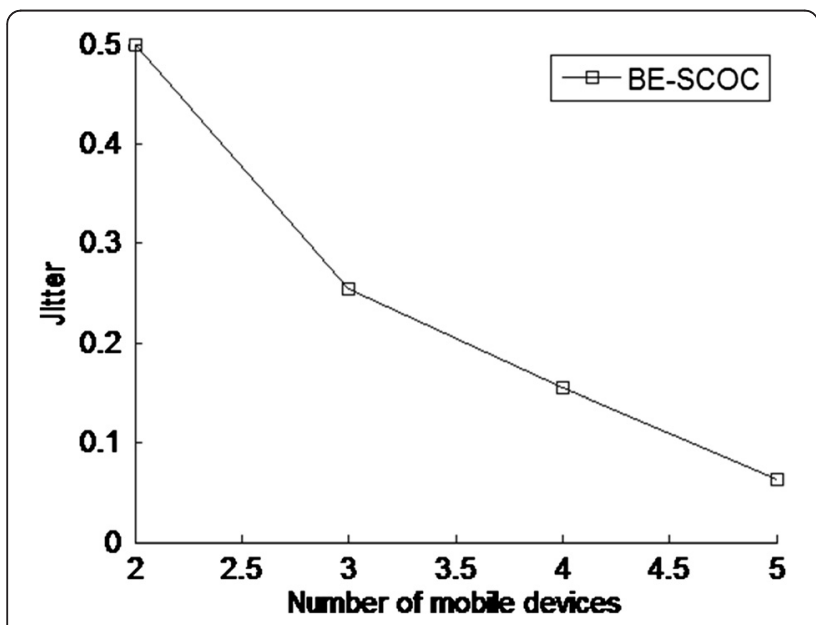

Fig. 5 Jitter

$$
\begin{aligned}
& \left\{\begin{array}{l}
f(y)=f\left(y_{i}, y_{j}\right) K_{B} \\
\Delta f\left(y_{t}, y_{t-k}\right)=G y(t)
\end{array}\right. \\
& G=\left[\begin{array}{cccc}
g_{1, t} & g_{2, t+1} & \cdots & g_{m, t+k} \\
\vdots & g_{2, t+2} & \cdots & \vdots \\
g_{t, t} & \ddots & \ddots & g_{t+k, t+m} \\
g_{m, n} & \cdots & \cdots & g_{m+k, m+k}
\end{array}\right] \\
& \left\{\begin{array}{c}
Y=\left[Y_{i}^{t}\left(y_{1}\right) Y_{i+1}^{t}\left(y_{2}\right) \cdots Y_{i+m}^{t}\left(y_{m}\right)\right]^{T} \\
O_{C}=G\left[K_{B}^{t}\left(Y_{1}\right) K_{B}^{t}\left(Y_{2}\right) \cdots K_{B}^{t}\left(Y_{m}\right)\right]^{T}
\end{array}\right.
\end{aligned}
$$

Here, the matrix $G$ is the weighting factor matrix. $\mathrm{O}_{C}$ is the control matrix.

On the basis of the above model and probability calculation, Fig. 3 shows the mobile crowd biological engineering optimization opportunities to control architecture. Among them, the architecture of different biological engineering reactions was taken into account in the top categories. The top of the next layer is used to distinguish between data sources and data types. The mobile crowd upper layer is used to provide interface parameter data and the measurement model. The middle layer also provides data for lower linear programming and non-linear characteristics.

\section{Performance evaluation}

To analyze and validate the proposed scheme in the biological engineering control effect, we designed two sets of biological experiments. In order to verify the proposed scheme to the performance of the control mechanism and performance of embedded devices, there were two crowds of biological experiments in different experimental environments. The two test crowds adopted different experimental material.

The first set of experiments was the glutamic acid fermentation as the core of biological engineering as the experimental object. A biological fermentation experiment tank was used to provide the same experiment environment, which is shown in Fig. 4. In the experiment, the embedded mobile devices are a one-way data transmission between the tanks. Mobile crowd module control is two-way between tanks with the experiment. The biological reaction process of glutamic acid was controlled by the opportunistic center control equipment.

Experimental parameters from five batches of field experimental data are shown in Table 1. Figure 5 is verified by increasing the embedded mobile terminals that can effectively improve the jitter of biological reaction engineering. In Fig. 6, the biological response data error is analyzed, the proposed BE-SCOC scheme can effectively guarantee the stability of the data. Figure 7 shows the proposed BE-SCOC scheme under different temperature conditions of biological response delay. Based on the big data driven and 


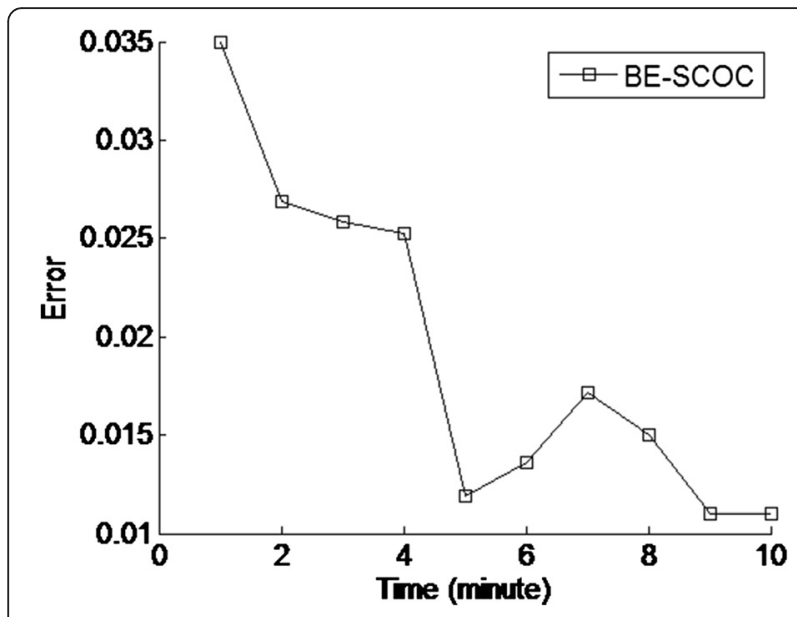

Fig. 6 Error

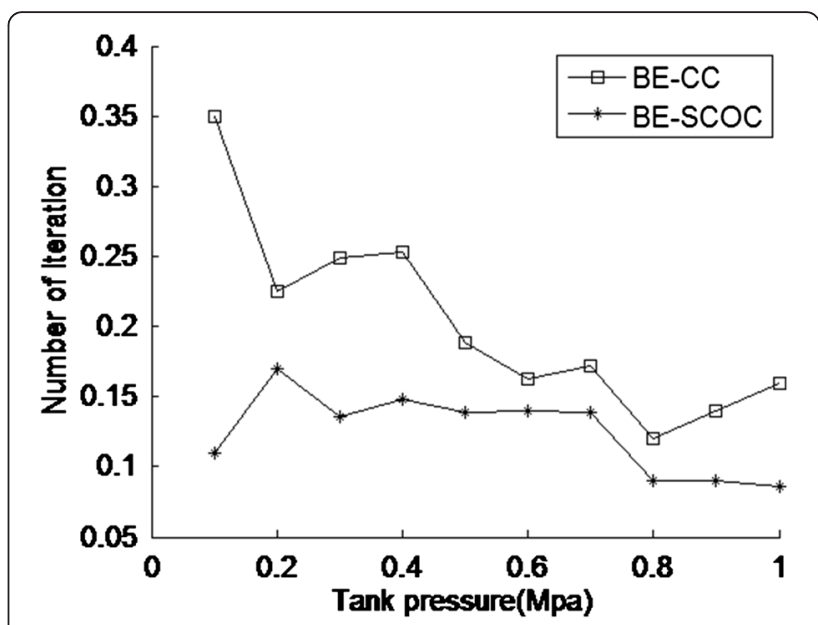

Fig. 8 Number of iterations opportunistic control, the glutamic acid fermentation reaction of the proposed scheme has the best performance. This control furthers optimization in mobile crowd biological engineering control.

In the second experiment, the iterations number of the proposed $\mathrm{BE}-\mathrm{SCOC}$ solution and the biological engineering control scheme based on the collaborative control (BE-CC) required were evaluated and studied in experiment 2 with the different air pressure environments. Figure 8 proves that the proposed $\mathrm{BE}-$ CC scheme is obviously better than the control optimization effect. It also benefited from embedded mobile terminals and the advantages of mobile crowd biological engineering.

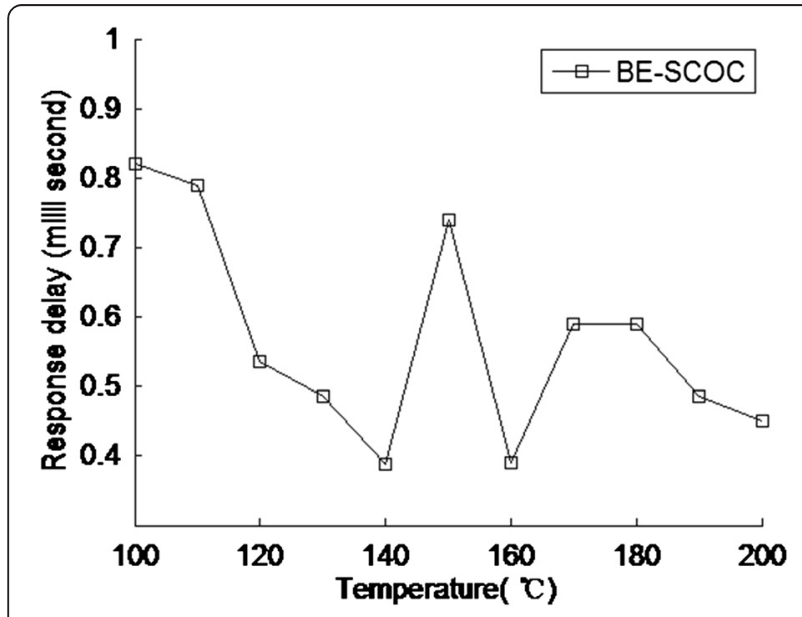

Fig. 7 Response delay

\section{Conclusions}

Large-scale biological engineering data increases the system control complexity. At the same time, there is a decrease in the mobile biological reaction control system performance. In order to solve the above problems, this paper puts forward a big data-driven and mobile crowd embedded chance control mechanism. On the one hand, the proposed data-driven larger embedded engineering measurement model can meet the demand of non-linear bioengineering and solve the problem of low accuracy. On the other hand, the embedded terminal actively eliminate random factors interference by sensing external interference factors. We proposed the mobile crowd biological engineering optimization opportunities control mechanism. Based on biological reaction time, experiment equipment size, and temperature of the experimental conditions, the established control mechanism can smooth the biological data jitter, reduce data error, and shorten the response delay. At the same time, the biological reaction scheme established by the convergence speed of pressure sensitivity is significantly lower than the biological engineering control scheme based on coordination control.

Acknowledgements

This work is supported in part by General projects supported by the natural science in Anhui Province Department of Education (KJ2014B186) and

Suzhou Institute of Characteristic Planting Seedling Production Engineering Technology Research Center Open Issues (2012YKF30).

Competing interests

The authors declare that they have no competing interests.

Received: 11 March 2016 Accepted: 13 May 2016

Published online: 01 June 2016

References

1. P Kruchten, The biological half-life of software engineering ideas. IEEE Softw. 25, 10-11 (2008) 
2. SJ Wu, CT Wu, JY Chang, Fuzzy-based self-interactive multiobjective evolution optimization for reverse engineering of biological networks. IEEE Trans. Fuzzy Syst. 20, 865-882 (2012)

3. College of Fellows, American Institute for Medical and Biological Engineering, Medical and biological engineering in the next 20 years: the promise and the challenges. IEEE Trans. Biomed. Eng. 60, 1767-1775 (2013)

4. Y Ning, C Multari, X Luo, C Palego, X Cheng, JCM Hwang et al., Broadband electrical detection of individual biological cells. IEEE Trans. Microwave Theory Tech. 62, 1905-1911 (2014)

5. D Leake, Next-gen gene synthesis enables large-scale engineering in biological systems: recent advances in synthetic biology are making this field more promising than ever. IEEE Pulse 6, 26-28 (2015)

6. MN Nounou, HN Nounou, N Meskin, A Datta, ER Dougherty, Multiscale denoising of biological data: a comparative analysis. IEEE/ACM Trans. Comput. Biol. Bioinform. 9, 1539-1545 (2012)

7. G Stegmayer, M Gerard, DH Milone, Data mining over biological datasets: an integrated approach based on computational intelligence. IEEE Comput. Intell. Mag. 7, 22-34 (2012)

8. AA Chatziioannou, I Kanaris, C Doukas, P Moulos, FN Kolisis, I Maglogiannis, GRISSOM platform: enabling distributed processing and management of biological data through fusion of grid and web technologies. IEEE Trans. Inf. Technol. Biomed. 15, 83-92 (2011)

9. H Nguyen, L Michel, JD Thompson, O Poch, Heterogeneous biological data integration with declarative query language. IBM J. Res. Dev. 58, 15:1-15:12 (2014)

10. S Carpendale, M Chen, D Evanko, N Gehlenborg, C Gorg, L Hunter et al., Ontologies in biological data visualization. IEEE Comput. Graph. Appl. 34, 815 (2014)

11. B Atakan, OB Akan, Biological foraging-inspired communication in intermittently connected mobile cognitive radio ad hoc networks. IEEE Trans. Veh. Technol. 61, 2651-2658 (2012)

12. J Lin, Wireless power transfer for cell phones or other mobile communication devices and biological implications [TCC Tidbits]. IEEE Microw. Mag. 14, 18-22 (2013)

13. X Liu, P Wang, Z Lan, S Binfei, Biological characteristic online identification technique over 5 G network. IEEE Wirel. Commun. 22, 84-90 (2015)

14. JC Lin, Wireless power transfer for mobile applications, and health effects. IEEE Antennas Propag. Mag. 55, 250-253 (2013)

15. MAI Tsompanas, GC Sirakoulis, Al Adamatzky, Evolving transport networks with cellular automata models inspired by slime mould. Cybern. IEEE Trans. 45, 1887-1899 (2015)

16. N Qian, Y Jiang, ZP Jiang, P Mazzoni, Movement duration, Fitts's law, and an infinite-horizon optimal feedback control model for biological motor systems. Neural Comput. 25, 697-724 (2013)

17. S Chowdhury, A Thakur, P Svec, C Wang, W Losert, SK Gupta, Automated manipulation of biological cells using gripper formations controlled by optical tweezers. IEEE Trans. Autom. Sci. Eng. 11, 338-347 (2014)

18. C Yao, X Zhang, F Guo, S Dong, FPGA-controlled all-solid-state nanosecond pulse generator for biological applications. IEEE Trans. Plasma Sci. 40, 23662372 (2012)

19. H Chen, C Wang, X Li, D Sun, Transportation of multiple biological cells through saturation-controlled optical tweezers in crowded microenvironments. IEEE/ASME Trans. Mechatron. 21, 1 (2015)

20. Y Okaie, AV Vasilakos, Transmission rate control for molecular communication among biological nanomachines. IEEE J. Sel. Areas Commun. 31, 835-846 (2013)

\section{Submit your manuscript to a SpringerOpen ${ }^{\circ}$ journal and benefit from:}

- Convenient online submission

- Rigorous peer review

- Immediate publication on acceptance

- Open access: articles freely available online

- High visibility within the field

Retaining the copyright to your article 